\title{
OCCULT PRIMARY BREAST CARCINOMA: A CASE REPORT
}

Mirella Laranjeira Nunes¹, Carlos Eduardo Caiado Anunciação², Vidianna Barbosa Sampaio², Rossano Robério Fernandes Araújo², Cinthya Roberta Santos de Jesus², Ana Leide Guerra dos Santos², Bruno Pacheco Pereira², João Esberard de Vasconcelos Beltrão Neto²

${ }^{1}$ Universidade de Pernambuco - Recife (PE), Brazil.

2Department of Mastology, Oswaldo Cruz University Hospital - Recife (PE), Brazil.

Introduction: Occult breast carcinoma $(\mathrm{OBC})$ is the histologically proven axillary lymph node (LN) metastasis, consistent with primary breast cancer, with no identifiable primary site. It is most commonly found in women above 60 years. Owing to the absence of protocols, management is challenging. According to the National Comprehensive Cancer Network (NCCN), the therapeutic options are mastectomy plus lymphadenectomy with or without radiotherapy, or lymphadenectomy with breast irradiation with or without axillary irradiation. Mastectomy is often used, but advances in neoadjuvant chemotherapy have made the survival between mastectomy and conservative breast management same. The prognosis is controversial, with lymph node (LN) involvement being the main factor. Case Report: A 45-year-old female presents with suspicious palpable right axillary lesion at level 1 topography of $2.5 \mathrm{~cm}$ size on the physical examination. No breast mass was palpable. Mammography was BIRADS classification 1. Breast and axillar ultrasound done 2 months before showed benign findings on the left side and axillary LN of $2.3 \mathrm{~cm}$ and breast nodule of $1.1 \mathrm{~cm} \times 0.9 \mathrm{~cm}$ on the right side. Core-needle biopsy showed fibroadenoma in the right-sided breast nodule and metastatic carcinoma in the axillary LN. Immunohistochemistry expression of the markers was consistent with breast origin and was progesterone and estrogen receptors positive and HER-2 negative. Magnetic resonance imaging (MRI) showed this atypical LN with $1.5 \mathrm{~cm}$. Clinical staging is T0N1M0. Neoadjuvant chemotherapy was performed with Adriamycin, cyclophosphamide, and paclitaxel. There was tumor remission with another MRI and ultrasonography showing the node metastasis with $1 \mathrm{~cm}$. Right radical mastectomy was performed. Anatomopathology showed cytoarchitectural changes due to chemotherapy, complete pathological response in the LN, and immunohistochemistry unchanged. In addition, tumorectomy were performed in the left-sided nodule, with anatomopathology showing ductal ectasia and histiocitary abscess. Radiotherapy at the supraclavicular area and tangents was performed, and tamoxifen was prescribed. The patient remained cancer free for 2 years after surgery.

Keywords: Occult Breast Cancer; Breast MRI; Breast Neoplasm. 